\title{
Scolia
}

Revue de linguistique

\section{Marie-José BÉGUELIN, Aidan CoveneY, Alexander GURYEV (éds), L'interrogative en français}

Hélène Vassiliadou

\section{OpenEdition \\ Journals}

Édition électronique

URL : http://journals.openedition.org/scolia/1021

DOI : 10.4000/scolia.1021

ISSN : 2677-4224

Éditeur

Presses universitaires de Strasbourg

Édition imprimée

Date de publication : 11 juillet 2019

Pagination : 180-184

ISBN : 979-1-03440-050-8

ISSN : 1253-9708

\section{Référence électronique}

Hélène Vassiliadou, « Marie-José Béguelin, Aidan coveney, Alexander Guryev (éds), L'interrogative en français », Scolia [En ligne], 33 | 2019, mis en ligne le 11 juillet 2019, consulté le 25 septembre 2020 URL : http://journals.openedition.org/scolia/1021 ; DOI : https://doi.org/10.4000/scolia.1021 
Marie-José BÉGUELIN, Aidan COVENEY, Alexander GURYEV (éds), L'interrogative en français, Berne, Peter Lang, collection Sciences pour la communication, 2018, 317 pages.

Les structures interrogatives du français intéressent depuis longtemps des chercheurs issus de domaines linguistiques différents, le nombre d'études récentes montre que cet engouement subsiste. Les éditeurs du volume 124 de la collection Sciences pour la communication réunissent les spécialistes de la question et livrent 11 articles et un avantpropos de haute qualité. La facture éditoriale est impeccable, exempte de coquilles. Tout en soulignant la variété formelle, sémantique et discursive du phénomène, Béguelin, Coveney \& Guryev intitulent l'ouvrage L'interrogative en français avec un singulier qui traduit la dimension holistique du sujet à travers une problématique commune qui lie la forme au sens. Il n'y a là rien de réducteur, l'éclectisme théorique se veut indispensable pour appréhender le domaine.

L'avant-propos (p. 7-18) fournit un état de l'art succinct focalisé sur les recherches récentes, une présentation des articles du volume et une bibliographie de deux pages. Les éditeurs livrent les raisons de l'intérêt des chercheurs pour les interrogatives et mettent en avant la richesse formelle des structures, lesquelles s'expliquent de manière différente selon la perspective théorique adoptée (facteurs inhérents à la langue, facteurs pragmatiques, caractère "polychronique» de la grammaire française (p.9), etc.). Les points de vue divergent en fonction des objectifs fixés: les approches formelles cherchent à élaborer des théories et des modèles syntaxiques en mettant à l'épreuve des exemples souvent fabriqués, alors que les approches plus variationnistes se concentrent sur la description de la profusion des structures formelles de la modalité interrogative. L'obstacle rencontré généralement dans les analyses concerne la difficulté à attribuer "stablement» (p. 8) à la forme interrogative une fonction sémantique de question, i.e. d'associer le concept de l'interrogation aux actes de langage signalés par cette opération.

Les articles, fondés sur la linguistique de corpus, font écho aux questions posées dans les débats théoriques évoqués dans l'avantpropos. Ils traitent des différences entre oral et écrit, du couplage entre intonation et fonction attribuée à telle ou telle structure intonative, 
de phénomènes plus spécifiques comme l'inversion interrogative ou le marquage par est-ce que, des différences entre interrogatives totales et partielles. On y trouve également des études contrastives (français/ italien ou anglais) et un intérêt manifeste pour la variation des structures en fonction du genre (bande dessinée, cinéma de banlieue, fables). Le spectre des sujets est large offrant une vue à la fois globale et pointue du domaine. Le compte rendu ne se prêtant pas à la revue de détail, nous soulignerons les principaux résultats des études sans respect de l'ordre d'apparition des contributions.

L'intonation donne du fil à retordre aux prosodistes qui s'accordent sur la difficulté à démontrer un parallélisme strict entre mélodie montante/ descendante et modalités assertive/ interrogative. L'article de Delais-Roussarie \& Herment (p. 51-78), à travers un corpus anglais/ français issu de tâches de lecture et/ou de parole authentique, se penche sur ces questions en évitant les généralisations fréquentes dans les manuels de FLE. Les auteures traitent les liens entre intonation et interprétation à la manière d'un «puzzle pluridimensionnel» en parcourant de manière exhaustive les types de phrases, d'actes et d'interprétation et en listant les paramètres nécessaires à l'étude de l'intonation des questions: traits grammaticaux, prosodiques, contours autres que terminaux, attaques, attitude et prise en charge du contenu propositionnel par le locuteur.

L'article de Druetta (p. 19-50) dresse un portrait typologique des tours interrogatifs et montre que dans le système français les différents types de marquage de l'interrogation n'ont rien d'original. L'article propose une description syntaxique homogène sans cliver l'écrit et l'oral. Il ressort également que, d'un point de vue typologique, les langues expriment préférentiellement l'interrogation par l'intonation. L'auteur parcourt de manière fine les paramètres de blocage et d'apparition des formes interrogatives du français via les notions de défectivité et de supplétivité. Druetta fournit une explication sur l'apparition de la forme en est-ce que qui en grammaticalisant le "dispositif de focalisation renfermant le marqueur VSc (= verbe sujet clitique) [...] pérennise la postposition du sujet clitique comme marque d'interrogation» (p. 25) sans perturber l'ordre de mots en SVO. Zumwald Küster (p. 95-118) examine aussi les raisons du figement de ce tour en diachronie et avance l'hypothèse que le français actuel possède deux formes est-ce que, l'une étant une particule unique 
(dispositif à inversion du sujet clitique) et l'autre étant constituée de trois morphèmes est, ce et que (dispositif de clivage). Qu'on accepte ou non cette position, ce travail a le mérite de pointer des phénomènes dépréciés par la norme et négligés dans les études offrant un éclairage particulier sur le fonctionnement de formes "dérivées» de est-ce que (cf. qu'est-ce que, c'est que, où est-ce que c'est que, etc.) employées pour la formation de questions partielles et plus rarement de questions totales.

Quant à l'inversion Verbe-sujet clitique (V-Scl), Berrendonner (p. 79-94) rejettel'hypothèse courante d'un simple marquage illocutoire. Selon lui, ce dispositif n'a pas pour rôle d'exprimer un acte de question: il est déclenché par la position non canonique d'un élément en tête de phrase, et est accompagné d'une emphase. L'inversion du sujet clitique a une signification modale, et non illocutoire exprimant l'indécision, l'incertitude et/ou l'éventualité. Une explication unifiée des phénomènes d'inversion est ainsi proposée permettant de justifier le déclin de l'usage de $\mathrm{V}$-Scl à partir du $\mathrm{xx}^{\mathrm{e}}$ siècle et la montée progressive de la structure in situ (cf. Tu vas où?), cette dernière se chargeant finalement d'exprimer l'acte de question.

Les études récentes explorant des corpus oraux valident cette dernière affirmation: la structure la plus sollicitée par les locuteurs est bien celle qui privilégie le maintien de l'ordre canonique SV pour les interrogatives partielles et totales. Guryev (p. 153-182) reprend et commente ces résultats, puis élargit le champ d'étude en analysant des échanges par SMS qui confirment que l'interrogation par SV ( $T u$ viens au cours?) domine. Le corpus SMS a la particularité de mélanger des formes jugées orales ou familières et des formes considérées comme soutenues. L'auteur suit une méthodologie minutieuse (annotation de corpus, exploitation des travaux antérieurs, précisions terminologiques, examen des contraintes qui régissent la sélection de variantes interrogatives), met en avant l'importance des "paramètres morphosyntaxiques dans la sélection des variantes de l'interrogation totale» (p. 158) et distingue des environnements morphosyntaxiques qui ont le pouvoir de déclencher une variabilité forte (cf. les verbes modaux) ou faible (cf. les configurations à arguments clitiques).

Ce volume sur l'interrogative a le mérite de présenter des résultats longitudinaux et de mettre en lumière l'évolution de la variation dans l'emploi des interrogatives. L'article de Dekhissi \& Coveney 
(p. 119-149), qui analyse quantitativement et en détail une masse de données importante issues d'un corpus filmique, arrive aussi à la conclusion que la structure in situ gagne du terrain. Il est fait état d'un inventaire de toutes les variantes présentes dans le corpus et d'un répertoire de facteurs qui conditionnent leur apparition. Les auteurs ont mis au jour des types de questions peu présentes dans des corpus plus conventionnels. Ainsi, ils mettent en avant l'essor de la question rhétorique dite conflictuelle en Qu'est-ce (que) (Qu'est-ce tu m'parles de mission?). Cette configuration syntaxique en déclin dans les questions ordinaires est remplacée par les formes en Comment!

L'interrogation rhétorique est aussi au centre de l'étude de Bonhomme (p. 247-269), qui analyse un corpus des Fables de la Fontaine. Selon lui, et à l'instar de certains prédécesseurs, il ne s'agit ni d'une "fausse question" ni d'une forme "réductible à une assertion» (p. 249). Il souligne en outre le fort potentiel énonciatif de l'interrogation rhétorique qui se manifeste à plusieurs niveaux: fonctionnel (dimension polyphonique et allocution d'un doute feint), interactif (dialogisme montré, fonction phatique), discursif (point de vue et prise en charge énonciative), sociodiscursif (éthos des locuteurs).

D'autres formes interrogatives sont examinées, les partielles averbales (A quand l'arrivée?) et surtout celles contenant ça et cela (Qui ça?/ Comment cela?) étudiées par Lefeuvre (p. 183-208), les constructions "préfacées par une interrogative» du type $\left[\mathrm{P}_{\mathrm{inv}}\right.$ ? $\left.\mathrm{Q}\right]$ comme dans Quelqu'un oserait-il devant moi dire du mal de la montagne $\mathrm{P}$, je le tuerais à l'instant même $\mathrm{Q}$ (p. 211) chez Corminboeuf (p. 209-227) ou encore les questions à valeur d'insinuation au conditionnel en français et au futur en italien (Serait-ce elle la coupable, o sarà invece lui ?) de Rossari (p. 229-246). Ces articles exposent différents effets discursifs déclenchés par ces structures, comme la facilitation du calcul de référence de la question grâce à ça/ cela, la formulation d'un argument faible en attente de "corroboration" à l'aide de questions alternatives ritualisées, l'ajout d'une valeur d'insinuation associée à des interrogatives que l'on présente comme une hypothèse.

Le dernier article du volume se différencie des autres en ce que Borel (p. 271-308) se penche sur un genre particulier, multimodal, la bande dessinée. Elle distingue les questions posées dans les récitatifs de celles rencontrées dans les bulles et examine leur portée (événements passés, présents ou à venir ou actions de personnages). L'auteure 
souligne la fonction de ces questions «dans l'économie narrative», leur poids dans le déroulement des aventures des personnages (surprises, rebondissements, incertitudes) et leur rôle dans l'introduction de nouvelles informations.

L'interrogative en français, tout en faisant la synthèse des acquis dans le domaine, offre à travers une quantité de données impressionnante les derniers résultats aussi bien en matière théorique qu'empirique. Les auteurs des articles procèdent avec rigueur scientifique et nous donnent les outils nécessaires pour une description précise de différents tours interrogatifs. On apprécie tout particulièrement le maniement de différentes dimensions mises en œuvre lors de l'analyse des exemples: prise en compte de paramètres syntaxiques et sémantiques mais aussi sociolinguistiques, pragmatiques et intonatifs, exploitation des analyses dites outillées, considération de genres discursifs et corpus variés, études diachroniques et contrastives. À maints égards, cet ouvrage constitue une source très utile d'informations. Nous en recommandons la lecture et l'exploitation. 